\title{
microRNA-21 protects against ischemia-reperfusion and hypoxia-reperfusion-induced cardiocyte apoptosis via the phosphatase and tensin homolog/Akt-dependent mechanism
}

\author{
QIONG YANG, KAN YANG and ANYING LI \\ Department of Cardiology, The Third Xiangya Hospital of Central South University, \\ Changsha, Hunan 410013, P.R. China
}

Received July 22, 2013; Accepted February 27, 2014

DOI: $10.3892 / \mathrm{mmr} .2014 .2068$

\begin{abstract}
Myocardial tissue injury caused by ischemia and hypoxia is a major cause of fatal diseases, including coronary atherosclerosis resulting from myocardial infarction and stroke. A number of microRNAs have been demonstrated to function as protectors against ischemia-reperfusion (I/R) and/or hypoxia-reperfusion (H/R)-induced myocardial injury, including microRNA-21 (miR-21). However, the protective mechanism of miR-21 has not been fully elucidated. The present study demonstrated that miR-21 had an anti-apoptotic role in I/R-induced myocardial damage in vivo and in $\mathrm{H} / \mathrm{R}$-induced $\mathrm{H} 9 \mathrm{C} 2$ cell death in vitro. Of note, the present study indicates that a common molecular mechanism is likely to exist in I/R- and H/R-induced cardiocyte apoptosis. During I/R and H/R, forced expression of miR-21 upregulated the Akt signaling activity via suppressing the expression of phosphatase and tensin homolog (PTEN) and the increased activity of Akt signaling further inhibited apoptosis partially by increasing the ratio of B-cell lymphoma 2(Bcl-2)/Bcl-2-associated X protein, which further suppressed the expression of caspase-3. In conclusion, to the best of our knowledge, it was shown for the first time that miR-21 had a protective role in I/R- and H/R-induced cardiocyte apoptosis via the PTEN/Akt-dependent mechanism. The present study indicates that miR-21 may be a promising agent for the treatment of I/R and H/R-induced myocardial injury.
\end{abstract}

\section{Introduction}

Tissue injury caused by ischemia or hypoxia is a major cause of fatal diseases, including coronary atherosclerosis caused by myocardial infarction and stroke $(1,2)$. The main causes of

Correspondence to: Professor Kan Yang, Department of Cardiology, The Third Xiangya Hospital of Central South University, 138 Tongzipo Road, Changsha, Hunan 410013, P.R. China E-mail: csuyangkan@163.com

Key words: hypoxia-reperfusion, microRNA-21, cardiocytes, ischemia-reperfusion, apoptosis myocardial ischemia injury include myocardial cell apoptosis, necrosis or temporarily impaired function, which are induced by myocardial hypoxia or/and lack of nutrients (3). In the treatment of ischemia, however, the restoration of blood supply may damage tissue, which is known as ischemia-reperfusion (I/R) injury.

The characteristics of I/R injury include apoptosis and necrosis of myocardial cells, the dysfunction of mitochondria, increase of lipid peroxides and the generation of free radicals $(4,5)$. For instance, free oxygen radicals, generated from the reaction between the oxygen carried by oxyhemoglobin in the blood and substances dissolved by impaired or necrotic myocardial cells caused by I/R, cause myocardial injury (6). Additionally, I/R injury also results in the inhibition of myocardial function, including the occurrence of malignant arrhythmia, the decrease of the left ventricular contractility and the decline of intraventricular pressure (7). As a result, developing an effective strategy for preventing and/or treating $\mathrm{I} / \mathrm{R}$ injury is urgently required.

microRNAs (miRNAs) are a group of endogenous, non-coding, single-strand, small RNAs of 22-25 nucleotides, which downregulate the expression of multiple target genes via degradation or translational inhibition of their mRNAs (8). According to statistics, miRNAs directly regulate $>30 \%$ of genes, which are associated with almost all major cellular functions, including cell growth, proliferation, differentiation, migration and apoptosis (9). It has been reported that several miRNAs have a crucial role in the protection against myocardial I/R injury (10). miR-21 has been found to be consistently upregulated in cardiac hypertrophy and to be relevant in the inhibition of cellular apoptosis (11). In fact, several targets of miR-21 have been demonstrated to be involved in the regulation of myocardial I/R injury, including phosphatase and tensin homolog (PTEN), programmed cell death 4 (PDCD4) and sprouty 1 and 2 (12).

PTEN is a negative regulator of Akt, which has a crucial role in cellular survival (13). B-cell lymphoma 2 (Bcl-2) and $\mathrm{Bcl}-2$-associated $\mathrm{X}$ protein (Bax), the representatives of the Bcl-2 family, are considered to be the primary regulators of apoptosis. Caspase- 3 is a downstream regulator of the Bcl-2 family and acts as a key effector of cellular apoptosis $(14,15)$. However, the anti-apoptotic mechanism of 
miR-21 in myocardial I/R injury has yet to be fully elucidated.

In the present study, the anti-apoptotic role of miR-21 in a rat model of myocardial I/R injury and in H9C2 cells with injury induced by hypoxia reoxygenation $(\mathrm{H} / \mathrm{R})$, was assessed by determining the expression of Bcl-2/Bax, caspase-3, PTEN and $\mathrm{p}-\mathrm{AKT}$.

\section{Materials and methods}

Reagents and materials. Dulbecco's modified Eagle medium (DMEM) was purchased from Gibco Laboratories (Grand Island, NY, USA). OPTI-minimal essential medium $\left(\mathrm{MEM}^{\circledR}\right)$, fetal bovine serum (FBS), TRIzol, TaqMan quantitative reverse transcription polymerase chain reaction (qRT-PCR) miRNA assay kit, RT-PCR kit, Lipofectamine 2000 , miR-21 mimics and miR-21 inhibitor were purchased from Thermo Fisher Scientific (Waltham, MA, USA). SYBR ${ }^{\circledR}$ Green qRCR mix was purchased from Toyobo Co., Ltd. (Osaka, Japan). All the antibodies were purchased from Abcam (Cambridge, UK).

Rat model of $I / R$ injury. All the protocols in the present study's experiment were approved by the Animal Ethics Committee of Central South University (Changsha, China). All Sprague-Dawley female rats (age, 10 weeks; weight, 250-300 g) were purchased from the Animal Center of Central South University. These rats were divided into four groups, including sham (served as controls), I/R 2, 4 and $6 \mathrm{~h}$. Under sterile conditions, intraperitoneal injection of $10 \%$ chloral hydrate $(350 \mathrm{mg} / \mathrm{kg})$ was performed. Following endotracheal intubation, a ventilator was used to support their lives. The heart was then exposed and the aorta was clamped with a non-invasive vascular clamp for $10 \mathrm{sec}$. Subsequent to that, the aorta was reperfused for 2,4 and $6 \mathrm{~h}$, respectively. In the sham group, the heart was exposed without clamping the aorta.

Adenovirus-mediated miR-21 gene transfer in vivo. To further investigate the role of miR-21 in myocardial I/R injury, the rAAV9-ZsGreen-pre-miR-21 adenovirus was constructed using the rAAV9-ZsGreen expression system (Clontech Laboratories, Mountain View, CA, USA) according to the manufacturer's instructions. The rAAV9-ZsGreen adenovirus was used as a negative control. The titer was $5.0 \times 10^{12} \mathrm{vg} / \mathrm{ml}$. In total, 30 rats were divided into five groups. In the control group, rats were injected with rAAV9-ZsGreen adenovirus through the coronary artery. In the miR-21 group, rats were injected with rAAV9-ZsGreen-pre-miR-21 adenovirus through coronary artery.In the sham group, rats were injected with rAAV9-ZsGreen adenovirus through the coronary artery, and 14 days following that, the sham-surgery was performed as described above. In the I/R group, rats were injected with rAAV9-ZsGreen adenovirus through the coronary artery, and 14 days after that, the I/R was performed as described above. In the $\mathrm{I} / \mathrm{R}+\mathrm{miR}-21$ group, rats were injected with rAAV9-ZsGreen-pre-miR-21 adenovirus through the coronary artery, and 14 days after that, I/R was performed. At $2 \mathrm{~h}$ after $\mathrm{I} / \mathrm{R}$, the animals were sacrificed.

Cell culture. The human H9C2 cell line was purchased from The Institute of Cell Biology at the Chinese Academy of Sciences (Shanghai, China). H9C2 cells were cultured in DMEM containing $10 \% \mathrm{FBS}$ and incubated at $37^{\circ} \mathrm{C}$ in a humidified incubator with $5 \% \mathrm{CO}_{2}$.

Apoptosis analysis. Flow cytometry was used to determine the cell apoptosis with the Annexin V-fluorescein isothiocyanate (FITC) apoptosis detection kit (Abcam). At $24 \mathrm{~h}$ post-transfection, the cells were harvested and washed with cold phosphate-buffered saline (PBS) twice. Following that, $10^{6}$ cells were resuspended in $200 \mu \mathrm{l}$ binding buffer, $10 \mu \mathrm{l}$ Annexin V-FITC and $5 \mu \mathrm{l}$ propidium iodide phycoerythrin were added, and cells were incubated in the dark for $30 \mathrm{~min}$. Next, $300 \mu \mathrm{l}$ binding buffer was added followed by flow cytometric analysis.

$H / R$ treatment of $\mathrm{H} 9 \mathrm{C} 2$ cells. H9C2 cells were cultured in DMEM with neither serum nor antibiotics at $37^{\circ} \mathrm{C}$ with $5 \% \mathrm{CO}_{2}$ for $12 \mathrm{~h}$, which were then cultured at $37^{\circ} \mathrm{C}$ with $1 \% \mathrm{O}_{2}-94 \% \mathrm{~N}_{2}-5 \% \mathrm{CO}_{2}$ for $4 \mathrm{~h}$. Subsequent to that, the cells were cultured in DMEM containing $10 \% \mathrm{FBS}$, incubated at $37^{\circ} \mathrm{C}$ with $5 \% \mathrm{CO}_{2}$ for $3 \mathrm{~h}$ and used in the following experiments.

In the in vitro experiment, $\mathrm{H} 9 \mathrm{C} 2$ cells were divided into the following groups: In the vehicle control (VC) group, the $\mathrm{H} 9 \mathrm{C} 2$ cells were without any treatment. In the inhibitor negative control (INC) group, the cells were transfected with $50 \mathrm{nM}$ miR-21 inhibitor. In the mimics NC (MNC) group, the cells were transfected with $50 \mathrm{nM} \mathrm{NC}$ mimics. In the $\mathrm{VC}+\mathrm{H} / \mathrm{R}$ group, the cells were treated with $\mathrm{H} / \mathrm{R}$. In the INC+H/R group, the cells transfected with $50 \mathrm{nM} \mathrm{NC}$ inhibitor were then treated with $\mathrm{H} / \mathrm{R}$. In the $\mathrm{MNC}+\mathrm{H} / \mathrm{R}$ group, the cells transfected with $50 \mathrm{nM} \mathrm{NC}$ mimics were then treated with $\mathrm{H} / \mathrm{R}$. In the miR-21 inhibitors $+\mathrm{H} / \mathrm{R}$ group, the cells transfected with $50 \mathrm{nM}$ miR-21 inhibitor were then treated with $\mathrm{H} / \mathrm{R}$. In the miR-21 mimics $+\mathrm{H} / \mathrm{R}$ group, cells transfected with $50 \mathrm{nM}$ miR-21 mimics were then treated with $\mathrm{H} / \mathrm{R}$.

$q P C R$ analysis. Total RNA was extracted with TRIzol according to the manufacturer's instructions. For miR-21 expression analysis, $2 \mu \mathrm{g}$ RNA was transcribed to cDNA using a stem-loop RT primer (Invitrogen Life Technologies, Carlsbad, CA, USA) and a miRNA reverse transcription kit (Applied Biosystems, Foster City, CA, USA) was used. The U6 gene was used as a normalization control. The amount of miR-21 to U6 was calculated using the equation $2^{-\Delta \mathrm{CT}}$, with $\Delta \mathrm{C}_{\mathrm{T}}=\mathrm{C}_{\mathrm{T}, \mathrm{miR}-21}-\mathrm{C}_{\mathrm{T}, \mathrm{U} 6}$.

For the detection of PTEN mRNA expression, qPCR analysis was performed using SYBR Green qPCR Mix and specific primers synthesized from Sangon Company (Shanghai, China). The following primers were used for amplification of PTEN: sense, 5'-GACGACAATCATGTTGCAGCA-3' and antisense, 5'-GCCTTTAAAAACTTGCCCCG-3'. GAPDH was used as an internal control with sense, 5'-ACAACTTTGGTATCGTGGAAGG-3' and antisense, 5'-GCCATCACGCCACAGTTTC-3'. The relative expression levels of genes were analyzed by the $2^{-\Delta \Delta C T}$ method.

Western blot analysis. Tissue samples were snap-frozen using liquid nitrogen in a mortar and vigorously ground. Cell samples were rinsed twice with cold PBS. Next, cold 
A

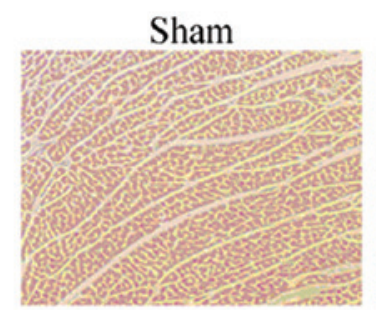

C
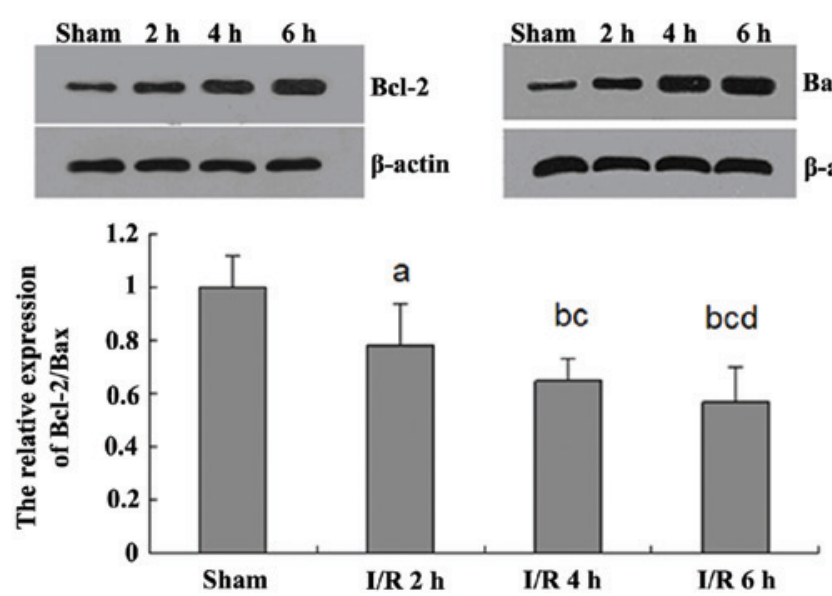

B

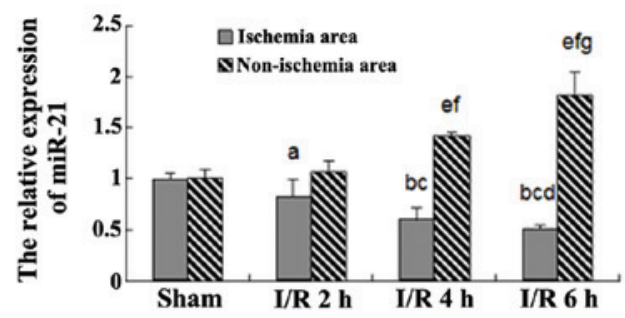

D

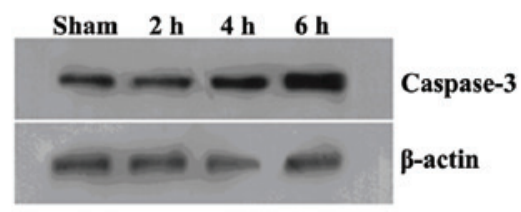

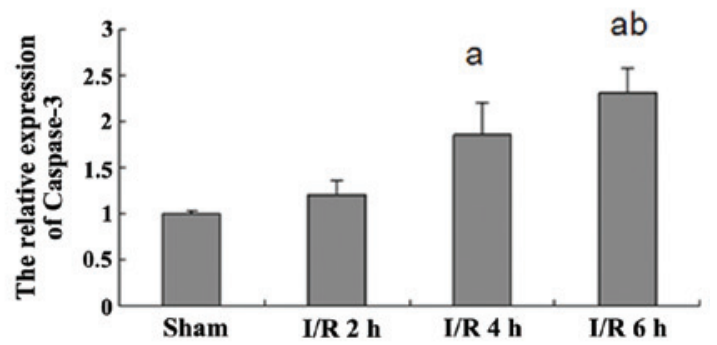

Figure 1. Expression of miR-21 in the myocardial tissue of rat model of I/R injury. (A) hematoxylin and eosin staining images of the myocardial tissues in the sham and I/R $2 \mathrm{~h}$ groups. (B) Quantitative polymerase chain reaction assay determined the relative expression of miR-21 in the ischemic and non-ischemic areas of the myocardial tissue in each group $\left({ }^{\mathrm{a}} \mathrm{P}<0.05\right.$ and ${ }^{\mathrm{b}} \mathrm{P}<0.01 \mathrm{vs}$. sham group; ${ }^{\mathrm{C}} \mathrm{P}<0.05 \mathrm{vs}$. I/R $2 \mathrm{~h}$ group; ${ }^{\mathrm{d}} \mathrm{P}<0.05 \mathrm{vs}$. I/R $4 \mathrm{~h}$ group; ${ }^{\mathrm{P}} \mathrm{P}<0.05 \mathrm{vs}$. sham group; ${ }^{\mathrm{f}} \mathrm{P}<0.05$ vs. I/R $2 \mathrm{~h}$ group; and ${ }^{\mathrm{g}} \mathrm{P}<0.05$ vs. I/R $4 \mathrm{~h}$ group). (C) Western blot analysis was used to determine the protein expression of Bcl- 2 and Bax in each group. $\beta$-actin was used as an internal reference. The relative expression of $\mathrm{Bcl}-2 / \mathrm{Bax}$ was calculated $\left({ }^{\mathrm{a}} \mathrm{P}<0.05\right.$ and ${ }^{\mathrm{b}} \mathrm{P}<0.01$ vs. sham group; ${ }^{\mathrm{c}} \mathrm{P}<0.05 \mathrm{vs}$. I/R $2 \mathrm{~h}$ group and ${ }^{\mathrm{d}} \mathrm{P}<0.05$ vs. I/R $4 \mathrm{~h}$ group). (D) Western blot analysis was used to determine the protein expression of caspase-3 in each group. $\beta$-actin was used as an internal reference ( $\left({ }^{\mathrm{P}}<0.01\right.$ vs. sham group and ${ }^{\mathrm{b}} \mathrm{P}<0.05$ vs. I/R 4 h group). I/R, ischemia-reperfusion; Bcl-2, B-cell lymphoma 2 ; Bax, Bcl-2-associated $\mathrm{X}$ protein; miR, microRNA.

radioimmunoprecipitation assay buffer was used to lyse the protein from the tissue or cell samples. The concentration of protein was determined using a bicinchoninic acid assay kit. Following that, proteins of $20 \mu \mathrm{g} /$ lane were loaded on a $10 \%$ SDS-PAGE to be separated, and then electrophoretically transferred to polyvinylidene fluoride membranes. Proteins on the membranes were then probed using primary antibodies, including mouse anti-Bcl-2, Bax, caspase-3, PTEN, p-Akt and $\beta$-actin, according to the manufacturer's instructions. Following incubation with secondary antibodies, including rabbit anti-mouse secondary antibody, the results were visualized with horseradish peroxidase and an enhanced chemiluminescence system, and quantified by the Quantity One software (Bio-Rad, Hercules, CA, USA).

Statistical analysis. Values are expressed as the mean \pm standard deviation of three independent experiments. A statistical analysis was performed by the SPSS 19.0 software (SPSS, Inc., Chicago, IL, USA). One-way analysis of variance and Student's t-test were used to analyze all the data. $\mathrm{P}<0.05$ was considered to indicate a statistically significant difference.

\section{Results}

Expression of miR-21 in the myocardial tissue of the rat model of $I / R$ injury. As shown in Fig. 1A, the myocardial tissue in the sham group demonstrated normal morphology, and its structure was clear without any edema or inflammatory cell infiltration. However, in the I/R $2 \mathrm{~h}$ group, the myocardial tissue exhibited structural disorder, irregular nuclei and edema. This result indicates that the rat model of I/R injury was successfully established.

miR-21 has been implicated to be involved in the myocardial I/R injury. Thus, qPCR was further applied in order to determine the expression levels of miR-21 in the ischemic and non-ischemic area of the myocardial tissue in each group. As demonstrated in Fig. 1B, in the ischemic area of the myocardial tissue, the expression levels of miR-21 exhibited a decreasing tendency with the extension of I/R time. However, in the non-ischemic area of the myocardial tissue, the miR-21 expression was gradually upregulated with the extension of I/R time. These data indicate that miR-21 may have an inhibitory role in the myocardial tissue injured by $\mathrm{I} / \mathrm{R}$.

Apoptotic pathways in response to $I / R$ injury. In order to gain a better understanding of the molecular mechanism underlying myocardial I/R injury, the present study focused on cellular apoptosis. The Bcl-2 family has a crucial role in the regulation of apoptosis. Thus, western blot analysis was applied to examine the protein expression levels of Bcl-2 and Bax, two key members in the Bcl-2 family. As shown in Fig. 1C, I/R injury increased the Bcl-2 and Bax protein expression levels in a time-dependent manner. However, the relative ratio of $\mathrm{Bcl}-2$ to $\mathrm{Bax}$ was gradually decreased, indicating that apoptotic signaling was activated. Additionally, the expression levels of caspase-3, a downstream effector 

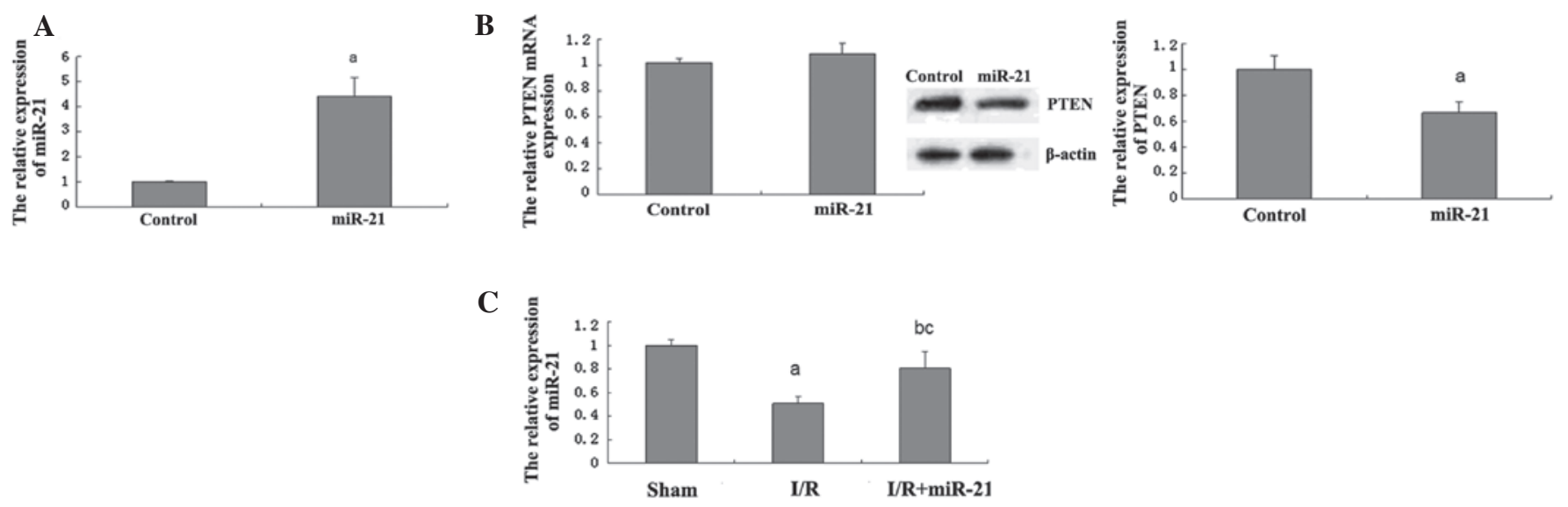

D

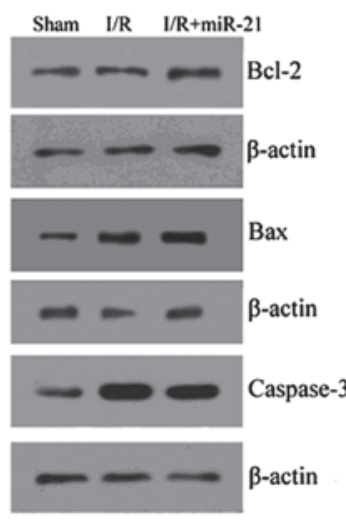

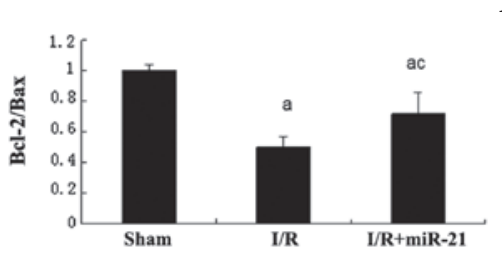

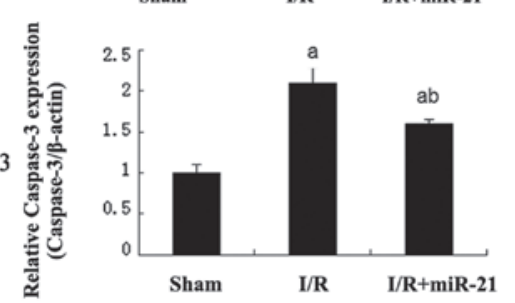

$\mathbf{E}$

Figure 2. Role of miR-21 in the early phase of myocardial I/R injury in rats. (A) The rAAV9-ZsGreen-pre-miR-21 (miR-21 group) or rAAV9-ZsGreen adenovirus (control group) was injected into the coronary artery of the rats, respectively. qPCR assay determined the relative expression of miR-21 following transfection. (a $<<0.05$ vs. control group). (B) Following injection mentioned above, the mRNA and protein expression of PTEN was examined by qPCR and western blot analysis, respectively. ( ${ }^{\mathrm{a}} \mathrm{P}<0.05$ vs. control group). (C) The expression of miR-21 was determined in each group. In the sham group, rats were injected with rAAV9-ZsGreen adenovirus through the coronary artery, and 14 days after that, the sham-operation was performed as described above. In the I/R group, rats were injected with rAAV9-ZsGreen adenovirus through the coronary artery, and 14 days after that, the I/R was performed as described above. In the I/R+miR-21 group, rats were injected with rAAV9-ZsGreen-pre-miR-21 adenovirus through the coronary artery, and 14 days after that, I/R was performed. ( ${ }^{\mathrm{a}} \mathrm{P}<0.01$ and ${ }^{\mathrm{b}} \mathrm{P}<0.05$ vs. sham group; ${ }^{\mathrm{C}} \mathrm{P}<0.05$ vs. I/R group). (D) Western blot analysis was then performed to determine the protein expression of $\mathrm{Bcl}-2, \mathrm{Bax}$ and caspase- 3 in each group. $\beta$-actin was used as an internal reference. The relative expression of Bcl $2 /$ Bax was calculated. $\left({ }^{\mathrm{a}} \mathrm{P}<0.01 \mathrm{vs}\right.$. sham group; ${ }^{\mathrm{b}} \mathrm{P}<0.01$ and ${ }^{\mathrm{C}} \mathrm{P}<0.05 \mathrm{vs.} \mathrm{I} / \mathrm{R}$ group). (E) Western blot analysis was then performed to determine the protein expression of PTEN and $\mathrm{p}$-Akt in each group. $\beta$-actin was used as an internal reference. ( ${ }^{\mathrm{P}} \mathrm{P}<0.01$ and ${ }^{\mathrm{b}} \mathrm{P}<0.05$ vs. sham group; ${ }^{\mathrm{C}} \mathrm{P}<0.05$ and ${ }^{\mathrm{d}} \mathrm{P}<0.01$ vs. I/R group). miR, microRNA; I/R, ischemia-reperfusion; $\mathrm{PTEN}$, phosphatase and tensin homolog; Bcl-2, B-cell lymphoma 2; Bax, Bcl-2-associated X protein; qPRC, quantitative polymerase chain reaction.

of Bcl-2, were further determined in each group. As shown in Fig. 1D, I/R increased the expression levels of caspase-3 in a time-dependent manner, further indicating that, with increasing I/R time, apoptosis was gradually upregulated.

The role of miR-21 in the early phase of myocardial $I / R$ injury in rats. To further investigate the role of miR-21 in myocardial I/R injury, the rAAV9-ZsGreen-pre-miR-21 or rAAV9-ZsGreen adenovirus was injected into the coronary artery of the rats in each group, respectively. The expression levels of miR-21 were initially determined, and it was found that in the miR-21 group, the expression of miR-21 in the myocardial region was significantly upregulated as compared with that in the control group (Fig. 2A), indicating that the rAAV9-ZsGreen-pre-miR-21 adenovirus was able to effectively express pre-miR-21 in vivo.

Since PTEN has been demonstrated to be a target of miR-21 and to have a crucial role in the regulation of cellular biological processes, the mRNA and protein expression levels of PTEN were determined next. As shown in Fig. 2B, the mRNA expression of PTEN demonstrated no difference between the control group and miR-21 group; however, the protein expression levels in the miR-21 group were significantly downregulated as compared with those in the control group, indicating that miR-21 has a post-transcriptional inhibitory effect on PTEN expression. Additionally, in the $\mathrm{I} / \mathrm{R}$ group, the expression of miR-21 was notably decreased as compared with that in the sham group, while following injection with rAAV9-ZsGreen-pre-miR-21 adenovirus, the expression of miR-21 was restored (Fig. 2C).

Based on these data, expression levels of certain significant factors associated with apoptosis, including Bcl-2, Bax and caspase-3, were determined further. As shown in Fig. 2D, the protein expression levels of Bcl-2 and Bax were increased in the I/R group compared with the sham group; however, the Bcl-2/Bax ratio was decreased, which were reverted in the I/R+miR-21 group. Additionally, the protein levels of caspase- 3 were also upregulated in the $I / R$ group as 
A

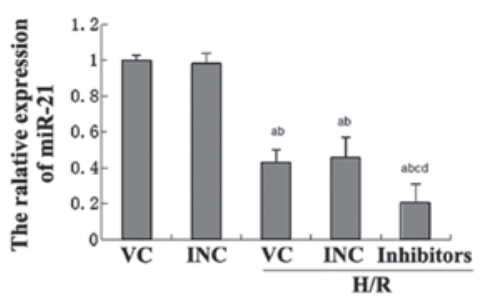

B

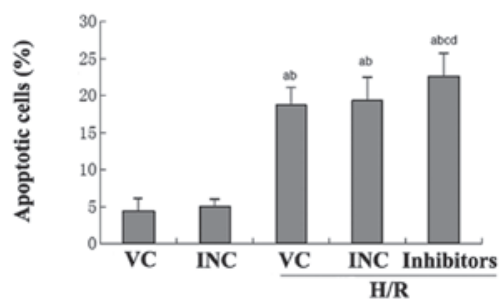

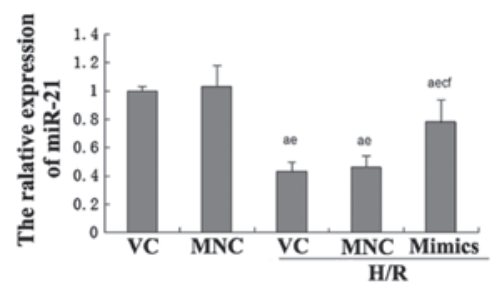

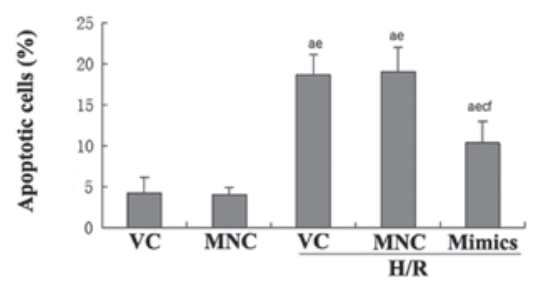

C
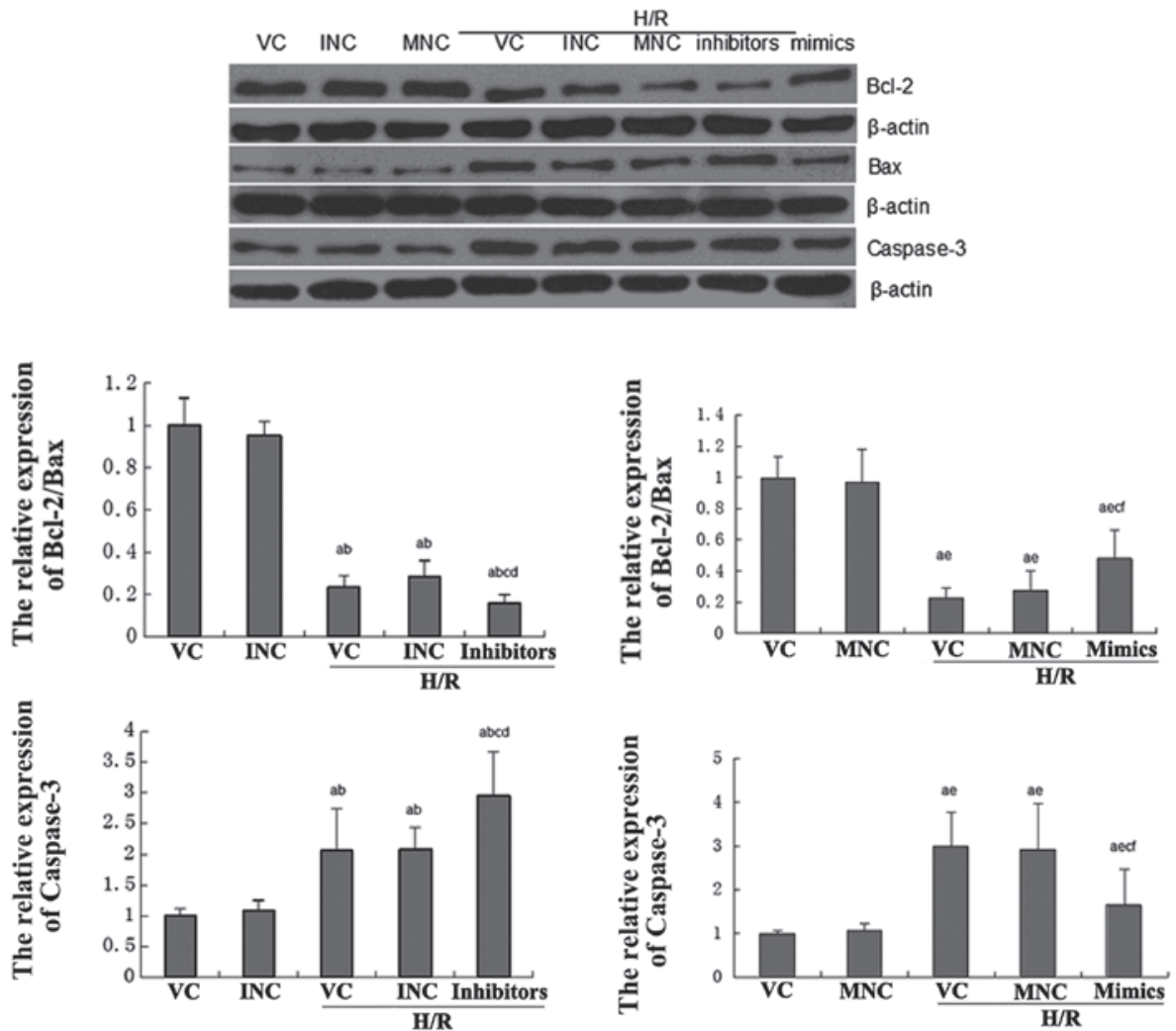

Figure 3. Role of miR-21 in H/R-induced apoptosis of $\mathrm{H} 9 \mathrm{C} 2$ cells. In the VC group, $\mathrm{H} 9 \mathrm{C} 2$ cells were without any treatment. In the INC group, cells were transfected with $50 \mathrm{nM}$ miR-21 inhibitor. In the MNC group, cells were transfected with $50 \mathrm{nM} \mathrm{NC}$ mimics. In the $\mathrm{VC}+\mathrm{H} / \mathrm{R}$ group, cells were treated with $\mathrm{H} / \mathrm{R}$. In the INC+H/R group, cells transfected with $50 \mathrm{nM} \mathrm{NC}$ inhibitor were then treated with $\mathrm{H} / \mathrm{R}$. In the MNC+H/R group, cells transfected with $50 \mathrm{nM}$ $\mathrm{NC}$ mimics were then treated with $\mathrm{H} / \mathrm{R}$. In the inhibitors $+\mathrm{H} / \mathrm{R}$ group, cells transfected with $50 \mathrm{nM}$ miR-21 inhibitor were then treated with $\mathrm{H} / \mathrm{R}$. In the mimics+H/R group, cells transfected with $50 \mathrm{nM}$ miR-21 mimics were then treated with $\mathrm{H} / \mathrm{R}$. (A) Quantitative polymerase chain reaction was performed to determine the expression of miR-21 in each group. (B) A cell apoptosis assay was performed in each group. (C) Western blot analysis was then performed to determine the protein expression of $\mathrm{Bcl}-2$, Bax and caspase-3 in each group. $\beta$-actin was used as an internal reference. The relative expression of Bcl2/Bax was calculated. H/R, hypoxia-reperfusion; miR, microRNA; Bcl-2, B-cell lymphoma 2; Bax, Bcl-2-associated X protein; NC, negative control; VC, vehicle control; INC, inhibitor negative control; MNC, minics NC; PTEN, phosphatase and tensin homolog.

compared with those in the sham group, which were reverted in the I/R+miR-21 group. These results indicate that miR-21 has an anti-apoptotic role in I/R-induced myocardial injury.

PTEN has an inhibitory role in the regulation of the Akt signaling pathway, which acts as a key regulator in cellular survival. Thus, to further investigate the involved regulatory mechanism, the expression of PTEN and the phosphorylation levels of Akt were determined, which directly reflect the activity of the Akt signaling pathway. As demonstrated in Fig. 2E, the expression of PTEN was significantly increased 


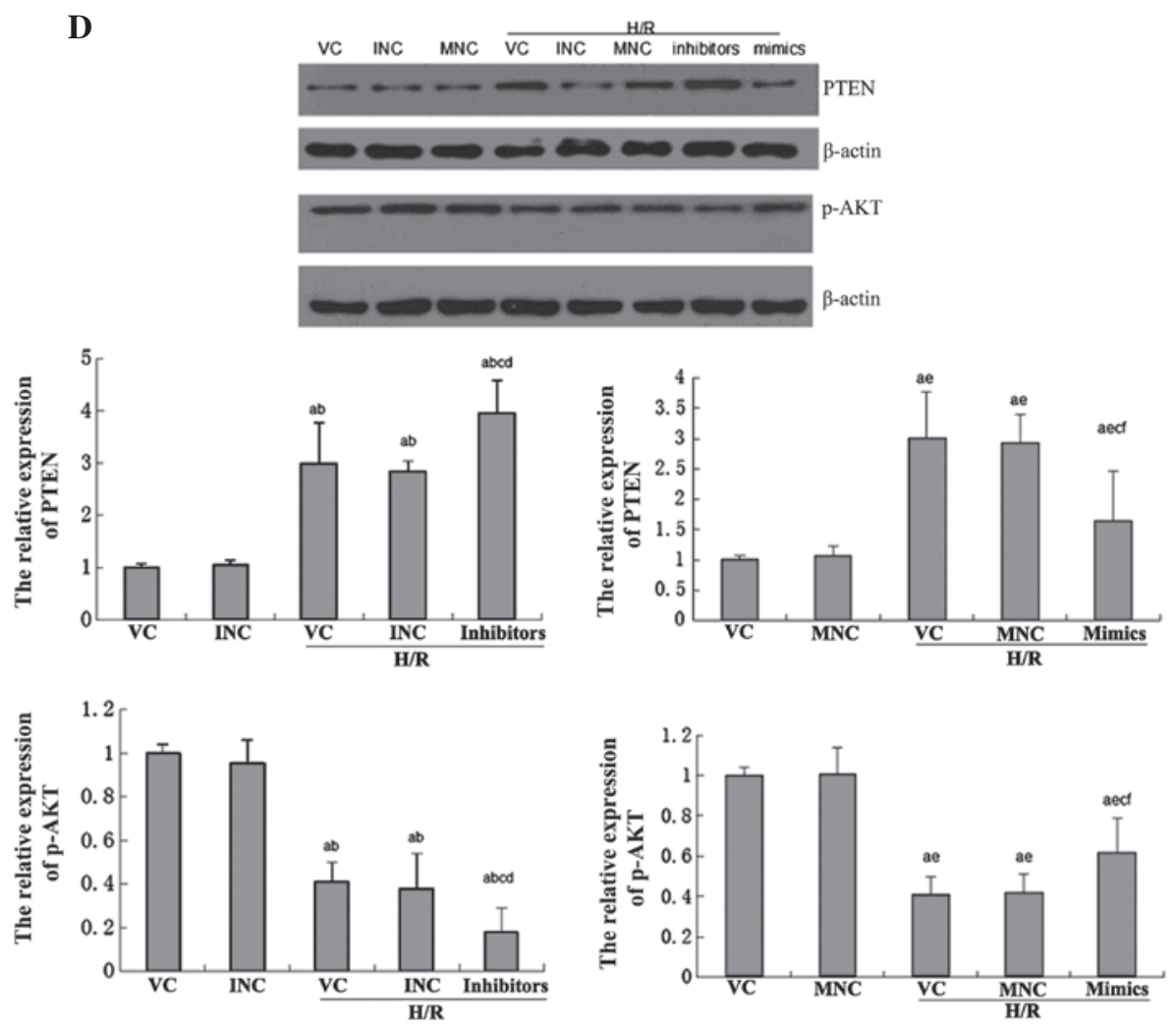

Figure 3. Continued. (D) Western blot analysis was then performed to determine the protein expression of PTEN and p-Akt in each group. $\beta$-actin was used as an internal reference. For Fig. 3 , ${ }^{\mathrm{a}} \mathrm{P}<0.01$ vs. VC group; ${ }^{\mathrm{b}} \mathrm{P}<0.01$ vs. INC group; ${ }^{\mathrm{C}} \mathrm{P}<0.05$ vs. $\mathrm{VC}+\mathrm{H} / \mathrm{R}$ group; ${ }^{\mathrm{d}} \mathrm{P}<0.05$ vs. INC+H/R group; ${ }^{\mathrm{e}} \mathrm{P}<0.05$ vs. $\mathrm{MNC}$ group; and ${ }^{\mathrm{f}} \mathrm{P}<0.05$ vs. MNC+H/R group. H/R, hypoxia-reperfusion; miR, microRNA; Bcl-2, B-cell lymphoma 2; Bax, Bcl-2-associated X protein; NC, negative control; VC, vehicle control; INC, inhibitor negative control; MNC, minics NC; PTEN, phosphatase and tensin homolog.

in the I/R group compared with the control group, which was, to a certain degree, reverted in the I/R+miR-21 group. Furthermore, as expected, the phosphorylation levels of Akt were evidently decreased in the I/R group compared with the control group, which could be restored in the I/R+miR-21 group. These findings indicate that miR-21 protects against I/R-induced myocardial cell apoptosis, most likely by inhibiting PTEN and therefore upregulating the activity of the Akt signaling pathway, which further suppresses pro-apoptotic factors such as caspase-3, while increasing anti-apoptotic factors, including $\mathrm{Bcl}-2 / \mathrm{Bax}$.

The role of miR-21 in H/R-induced apoptosis of H9C2 cells. The cardiac myoblast cell line H9C2 was used to investigate the role of miR-21 in H/R-induced cellular apoptosis. The expression of miR-21 in each group was initially determined, and $H / R$ treatment and an miR-21 inhibitor were found to be capable of significantly downregulating the expression of miR-21, which was restored by miR-21 mimics, as expected (Fig. 3A). Subsequent to that, the apoptotic levels in each group were examined. As shown in Fig. 3B, induction of H/R and presence of the miR-21 inhibitor significantly enhanced cellular apoptosis, which was restored by miR-21 mimics.

To further investigate the molecular mechanisms involved, the protein levels of Bcl-2, Bax and caspase-3 were examined in each group. As shown in Fig. 3C, H/R treatment and presence of the miR-21 inhibitor significantly downregulated the ratio of $\mathrm{Bcl}-2 / \mathrm{Bax}$ while increasing the expression of caspase-3, which was also reverted by the miR-21 mimics.
The role of miR-21 in H/R-induced activation of PTEN/Akt signaling. Consistent with the aforementioned results in I/R-induced myocardial injury experiments, it was also identified that $\mathrm{H} / \mathrm{R}$ treatment and miR-21 inhibitor evidently upregulated the PTEN expression while reducing the phosphorylation levels of Akt, which was also reverted by miR-21 mimics (Fig. 3D).

In summary, the results indicate that miR-21 has an inhibitory role in H/R-induced cellular apoptosis, partially by inhibiting PTEN expression and thus promoting the activity of the Akt signaling pathway, which further suppresses the expression levels of caspase-3 while increasing the protein ratio of $\mathrm{Bcl}-2 / \mathrm{Bax}$.

\section{Discussion}

The present study found that miR-21 had an anti-apoptotic role in I/R-induced myocardial damage in vivo, and in $\mathrm{H} / \mathrm{R}$-induced H9C2 cell death in vitro. Of note, the present study indicated that a common molecular mechanism is likely to exist in I/Rand H/R-induced cardiocyte apoptosis, and that during I/R and $\mathrm{H} / \mathrm{R}$, miR-21 can upregulate the Akt signaling activity via suppressing the expression of PTEN. This increased activity of Akt signaling further inhibits cell apoptosis, partially by upregulating the ratio of $\mathrm{Bcl}-2 / \mathrm{Bax}$, which can reduce the expression of caspase- 3 .

Thus far, the biological role of miR-21 in cardiocytes has not been fully elucidated. Cheng et al (16) have reported that miR-21 is highly expressed in the adult heart, indicating that it 
may have a crucial role in the regulation of normal biological functions of the myocardial tissue. Previously, accumulating evidence has shown that miR-21 has a protective effect on cardiocyte apoptosis via its target genes. For example, Qin et al (17) revealed that miR-21 inhibited left ventricular remodeling in the early phase of I/R injury by suppressing cell apoptosis in rats. Sayed et al (18) reported that Akt mediated the anti-apoptotic effects of miR-21 via suppression of the Fas ligand. Besides, miR-21 has been found to protect against the $\mathrm{H}_{2} \mathrm{O}_{2}$-induced myocardial cell injury via targeting PDCD4 (19). In the present study, it was revealed that the PTEN/Akt dependent mechanism involved in I/R- and H/R-induced cardiocyte apoptosis in vivo or in vitro, respectively. In fact, PTEN has previously been shown to be a direct target of miR-21 $(20,21)$, and downregulate the regulation of Akt signaling, which has a crucial effect on the cell survival rate (22). Recently, it has also been reported that miR-21 protects cardiomyocytes from tumor necrosis factor- $\alpha$-induced apoptosis in vitro via modulating the PTEN/Akt/forkhead box O3A pathway (23).

It was further revealed that the protein expression levels of several key apoptotic effectors, including Bcl-2, Bax and caspase-3, were mediated by miR-21 in rat and cell models of I/R or H/R injury, respectively. Bcl-2 is a highly conserved anti-apoptotic protein in the $\mathrm{Bcl}-2$ family and has a low expression or no expression in apoptotic cells. A number of studies have indicated that $\mathrm{Bcl}-2$, together with several mitochondrial membrane-associated proteins, can suppress the production of free radicals and thus inhibit apoptosis via downregulating cell endoplasmic reticulum $\mathrm{Ca}^{2+}$ release or the formation of lipid peroxides. Of note, $\mathrm{Bcl}-2$ has been suggested to have a central role in the promotion of cardiomyocyte survival by suppressing apoptosis (24). It has been well established that Bax, generally expressed in the majority of tissues and organs, has a pro-apoptotic role in the mitochondrial-dependent apoptotic pathway (25). In fact, Bax exerts its pro-apoptotic role by inhibiting the function of $\mathrm{Bcl}-2$ through a related protein homologue to $\mathrm{Bcl}-2$. Thus, Bax is a major endogenous antagonist of Bcl-2. On the contrary, however, Bcl-2 and B-cell lymphoma-extra large can form a heterodimer and cause Bax to lose its pro-apoptotic effect $(26,27)$. Thus, under physiological conditions, the expression of Bcl-2 and Bax are maintained on a balanced level, which, once broken, may induce cellular apoptosis.

Caspases are a significant cysteine-aspartate-specific protease family, ubiquitously expressed in various mammalian cells. It has been demonstrated that the activation of the caspase family acts as a key effector as well as the ultimate enforcer of cell apoptosis (28). Caspase-3 is a significant member of this protease family and its activation has been found in multiple types of cells undergoing apoptosis (29). Of note, $\mathrm{Bcl}-2$ and caspase- 3 have an interaction mechanism. Bcl-2 was previously found to be upstream of caspase-3, and to have an inhibitory role in the regulation of caspase-3 expression. Bcl-2 was then found to be a direct substrate of caspase-3, and thus, to be inversely regulated by caspase-3. Once hydrolyzed by caspase-3, the fragment of Bcl-2 is not likely to have any more anti-apoptotic function, but it was demonstrated to have pro-apoptotic activity $(15,30,31)$. As a result, there also exists a balance between Bcl-2 and caspase-3.

In conclusion, to the best of our knowledge, the present study was the first to reveal that miR-21 had a protective role in I/R- and H/R-induced cardiocyte apoptosis, most likely depending on a common mechanism, which is involved in the PTEN/Akt signaling activity, Bcl-2 protein family and caspase-3. As a result, it is speculated that miR-21 may be a promising agent for the treatment of I/R- and H/R-induced myocardial injury.

\section{References}

1. Ganguly R, Lytwyn MS and Pierce GN: Differential effects of trans and polyunsaturated fatty acids on ischemia/reperfusion injury and its associated cardiovascular disease states. Curr Pharm Des: Apr 10, 2013 (Epub ahead of print).

2. Kalogeris T, Baines CP, Krenz M and Korthuis RJ: Cell biology of ischemia/reperfusion injury. Int Rev Cell Mol Biol 298: 229-317, 2012.

3. $\mathrm{Xu} \mathrm{T}, \mathrm{Li} \mathrm{D}$ and Jiang D: Targeting cell signaling and apoptotic pathways by luteolin: cardioprotective role in rat cardiomyocytes following ischemia/reperfusion. Nutrients 4: 2008-2019, 2012.

4. Guo W, Kan JT, Cheng ZY, et al: Hydrogen sulfide as an endogenous modulator in mitochondria and mitochondria dysfunction. Oxid Med Cell Longev 2012: 878052, 2012.

5. Ha T, Liu L, Kelley J, Kao R, Williams D and Li C: Toll-like receptors: new players in myocardial ischemia/reperfusion injury. Antioxid Redox Signal 15: 1875-1893, 2011.

6. Young RW: Hyperoxia: a review of the risks and benefits in adult cardiac surgery. J Extra Corpor Technol 44: 241-249, 2012.

7. Nagai T, Anzai T, Kaneko H, et al: Impact of systemic acidosis on the development of malignant ventricular arrhythmias after reperfusion therapy for ST-elevation myocardial infarction. Circ J 74: 1808-1814, 2010.

8. Porrello ER: microRNAs in cardiac development and regeneration. Clin Sci (Lond) 125: 151-166, 2013.

9. Chen LJ, Lim SH, Yeh YT, Lien SC and Chiu JJ: Roles of microRNAs in atherosclerosis and restenosis. J Biomed Sci 19: 79, 2012.

10. Zhu $\mathrm{H}$ and Fan GC: Role of microRNAs in the reperfused myocardium towards post-infarct remodelling. Cardiovasc Res 94: 284-292, 2012.

11. Yang $\mathrm{KC}, \mathrm{Ku}$ YC, Lovett $\mathrm{M}$ and Nerbonne JM: Combined deep microRNA and mRNA sequencing identifies protective transcriptomal signature of enhanced PI3Kalpha signaling in cardiac hypertrophy. J Mol Cell Cardiol 53: 101-112, 2012.

12. Cheng $\mathrm{Y}$ and Zhang $\mathrm{C}$ : MicroRNA-21 in cardiovascular disease. J Cardiovasc Transl Res 3: 251-255, 2010.

13. Hers I, Vincent EE and Tavare JM: Akt signalling in health and disease. Cell Signal 23: 1515-1527, 2011.

14. Tomek M, Akiyama T and Dass CR: Role of Bcl-2 in tumour cell survival and implications for pharmacotherapy. J Pharm Pharmacol 64: 1695-1702, 2012.

15. Zakeri Z and Lockshin RA: Cell death: history and future. Adv Exp Med Biol 615: 1-11, 2008.

16. Cheng Y, Ji R, Yue J, et al: MicroRNAs are aberrantly expressed in hypertrophic heart: do they play a role in cardiac hypertrophy? Am J Pathol 170: 1831-1840, 2007.

17. Qin Y, Yu Y, Dong H, Bian X, Guo X and Dong S: MicroRNA 21 inhibits left ventricular remodeling in the early phase of rat model with ischemia-reperfusion injury by suppressing cell apoptosis. Int J Med Sci 9: 413-423, 2012.

18. Sayed D, He M, Hong C, et al: MicroRNA-21 is a downstream effector of AKT that mediates its antiapoptotic effects via suppression of Fas ligand. J Biol Chem 285: 20281-20290, 2010.

19. Cheng Y, Liu X, Zhang S, Lin Y, Yang J and Zhang C: MicroRNA-21 protects against the $\mathrm{H}(2) \mathrm{O}(2)$-induced injury on cardiac myocytes via its target gene PDCD4. J Mol Cell Cardiol 47: 5-14, 2009.

20. Liu CZ, Liu W, Zheng Y, et al: PTEN and PDCD4 are bona fide targets of microRNA-21 in human cholangiocarcinoma. Chin Med Sci J 27: 65-72, 2012.

21. Meng F, Henson R, Wehbe-Janek H, Ghoshal K, Jacob ST and Patel T: MicroRNA-21 regulates expression of the PTEN tumor suppressor gene in human hepatocellular cancer. Gastroenterology 133: 647-658, 2007. 
22. McCubrey JA, Steelman LS, Chappell WH, et al: Ras/Raf/MEK/ERK and PI3K/PTEN/Akt/mTOR cascade inhibitors: how mutations can result in therapy resistance and how to overcome resistance. Oncotarget 3: 1068-1111, 2012.

23. Tang Y and Wang MH: MicroRNA-21 protects cardiomyocytes from tumor necrosis factor-alpha induced apoptosis in vitro via modulating PTEN/AKT/FOXO3a pathway. Chinese Journal of Cardiovascular Diseases 41: 135-142, 2013 (In Chinese).

24. Ma YX, Guo Z and Sun T: CGRP inhibits norepinephrine induced apoptosis with restoration of $\mathrm{Bcl}-2 / \mathrm{Bax}$ in cultured cardiomyocytes of rat. Neurosci Lett 549: 130-134, 2013.

25. Xu XP, Zhai D, Kim E, et al: Three-dimensional structure of Bax-mediated pores in membrane bilayers. Cell Death Dis 4 e683, 2013.
26. Walensky LD and Gavathiotis E: BAX unleashed: the biochemical transformation of an inactive cytosolic monomer into a toxic mitochondrial pore. Trends Biochem Sci 36: 642-652, 2011.

27. Renault TT and Manon S: Bax: Addressed to kill. Biochimie 93: 1379-1391, 2011.

28. Fiandalo MV and Kyprianou N: Caspase control: protagonists of cancer cell apoptosis. Exp Oncol 34: 165-175, 2012.

29. Snigdha S, Smith ED, Prieto GA and Cotman CW: Caspase-3 activation as a bifurcation point between plasticity and cell death. Neurosci Bull 28: 14-24, 2012.

30. Poreba M, Strózyk A, Salvesen GS and Drag M: Caspase substrates and inhibitors. Cold Spring Harb Perspect Biol 5: a008680, 2013.

31. Kale J, Liu Q, Leber B and Andrews DW: Shedding light on apoptosis at subcellular membranes. Cell 151: 1179-1184, 2012. 